\title{
A quantitative cross-sectional survey of psychosocial stimulation and counselling interventions at nutritional rehabilitation units in Southern Malawi
}

\author{
Allison I Daniel ${ }^{1-3}$, Kate Chidzalo ${ }^{4,5}$, Isabel Potani ${ }^{3,4,5}$, Wieger Voskuij1 ${ }^{5,6}$, Melissa Gladstone ${ }^{7}$, Meta \\ van den Heuvel ${ }^{1,5,8,9}$, Mike Bwanali ${ }^{4,5}$, Emmie Mbale ${ }^{4,5}$, Robert H J Bandsma ${ }^{1-3,5,10}$ \\ 1. Centre for Global Child Health, Hospital for Sick Children, Toronto, Ontario, Canada \\ 2. Translational Medicine Program \\ 3. Department of Nutritional Sciences, Faculty of Medicine, University of Toronto, Toronto, Ontario, Canada \\ 4. Department of Paediatrics, College of Medicine, University of Malawi, Blantyre, Malawi \\ 5. The Childhood Acute Illness \& Nutrition Network (CHAIN), Blantyre, Malawi \\ 6. Global Child Health Group, Emma Children's Hospital, Amsterdam University Medical Centre, University of Amsterdam, Amsterdam, The \\ Netherlands \\ 7. Department of Women's and Children's Health, Institute of Translational Medicine, University of Liverpool, Liverpool, United Kingdom \\ 8. Division of Paediatric Medicine, Hospital for Sick Children, Toronto, Ontario, Canada \\ 9. Department of Paediatrics, Faculty of Medicine, University of Toronto, Toronto, Ontario Canada \\ 10. Department of Biomedical Sciences, College of Medicine, University of Malawi, Blantyre, Malawi
}

\section{Background}

Abstract

Inpatient treatment at nutritional rehabilitation units (NRUs) is needed for children who have severe acute malnutrition (SAM) and acute illness, loss of appetite, or severe oedema. World Health Organization guidelines state that nutritional counselling should be done with primary caregivers at NRUs. These recommendations also include psychosocial stimulation interventions to improve developmental outcomes in children with SAM. However, there is limited information about the delivery of these types of interventions for caregivers and children in NRU settings. The primary objective of this research was therefore to obtain data about NRU resources, activities, and protocols relevant to psychosocial stimulation and counselling interventions during inpatient treatment of children with SAM.

Methods

A cross-sectional survey was administered by interview at all 16 NRUs in seven districts in Southern Malawi. Participants were health workers, nurses, and nutritionists employed at the respective NRUs.

Results

The response rate was $100 \%$ across NRUs. Half of participants said that psychosocial stimulation interventions are conducted at their respective NRUs, yet none of the NRUs have protocols for delivery of these interventions. Furthermore, 7/16 (44\%) NRUs have no resources for psychosocial stimulation including play materials. Thirteen of $16(81 \%)$ participants said that they feel this type of intervention is very important and 3/16 (19\%) participants said that this somewhat important for children with SAM. All NRUs provide counselling to caregivers about breastfeeding and nutrition; 15/16 (94\%) also give counselling about water, sanitation and hygiene.

Conclusions

Ultimately, results from this survey highlighted that there is a need to invest in comprehensive interventions to improve developmental and nutritional outcomes in these vulnerable children requiring admission to NRUs.

Key words: severe acute malnutrition (SAM); child development; psychosocial stimulation; nutritional rehabilitation unit (NRU); Community-based Management of Acute Malnutrition (CMAM)

\section{Introduction}

Severe acute malnutrition (SAM), defined by severe wasting or oedematous malnutrition, remains highly prevalent in many parts of the world. Globally, an estimated 17 million children had SAM in the form of severe wasting in $2017^{1}$. The Community-based Management of Acute Malnutrition (CMAM) approach was introduced in low- and middleincome countries almost two decades ago, drastically changing the way that acute malnutrition is managed ${ }^{2}$. Its purpose is to identify acutely malnourished children and to provide appropriate nutritional care based on the severity of malnutrition and presence or absence of acute illnesses ${ }^{2}$. Children with SAM and acute illnesses, loss of appetite, or other complications require admission to nutritional rehabilitation units (NRUs) for more intensive clinical care and nutritional support ${ }^{2-4}$. For children with SAM without an acute illness or appetite loss, referral to outpatient therapeutic feeding programmes for provision of Ready-touse Therapeutic Food and regular monitoring of weight over time is needed ${ }^{2,3}$.

Malawi has historically had a high burden of malnutrition, though rates of acute malnutrition have steadily decreased from $7 \%$ in 2000 to $3 \%$ in $2015^{5}$. CMAM was first established in 2002 in Malawi as a pilot program until all districts in the country implemented CMAM by $2010^{6-8}$. There are currently 104 operational NRUs in Malawi for inpatient treatment of SAM $^{9}$. According to the 2016 Malawi CMAM guidelines, nurses are responsible for providing clinical and nutritional treatment at NRUs ${ }^{10}$. Health workers, known as homecraft workers in Malawi, are required to deliver health and nutrition counselling sessions to caregivers each day during children's inpatient treatment according to national guidelines ${ }^{10}$. 
Homecraft workers are engaged to assist caregivers with caring for their children, including feeding children therapeutic feeds, as well as playing with their children with the use of play materials that are intended to be available in NRUs ${ }^{10}$. This aligns with World Health Organization (WHO) guidelines for providing a stimulating environment for children in NRU settings ${ }^{11,12}$. According to the Ministry of Health in Malawi, nurses and homecraft workers are to counsel caregivers and give psychosocial support ${ }^{10-12}$.

Integrated interventions that include nutrition and water, sanitation and hygiene (WASH) interventions, promotion of responsive caregiving and psychosocial stimulation, and support for caregivers can promote better nutritional status and development in all children ${ }^{13-19}$. This is particularly relevant for children with SAM who have been shown to have poor developmental and nutritional outcomes, as well as high risk of mortality, after inpatient treatment at NRUs ${ }^{20,24}$. WHO guidelines emphasize that counselling, psychosocial stimulation, and caregiver support should be provided to children admitted to $\mathrm{NRUs}^{4,11}$. The recommendation for psychosocial stimulation is based on limited research showing improved developmental outcomes in children hospitalized with SAM in Jamaica and Bangladesh who received this type of intervention ${ }^{21,25,26}$. However, there is lacking information about how best to deliver these interventions for children with SAM. ${ }^{25}$ There has also been very limited research examining the extent to which the basic WHO recommendations, including counselling of caregivers and psychosocial stimulation for children with SAM, are considered and implemented in NRUs in Malawi and elsewhere ${ }^{12,27}$.

The overall aim of this research was therefore to evaluate NRU resources, activities, and protocols relevant to psychosocial stimulation and counselling interventions during inpatient treatment for SAM. An additional objective was to gain feedback about the importance of psychosocial stimulation interventions in NRU settings. This information will allow for a better understanding of existing programs at NRUs, areas of improvement, and potential for additional interventions.

\section{Methods}

A cross-sectional survey was administered through an inperson interview by a trained enumerator between October 16 and November 2, 2018 at all 16 NRUs in western districts in the Southern Region of Malawi (Table 1). The survey covered a 15,000 square kilometer radius inhabited by one-fifth of the population of Malawi, including all of the southwest part of the country (Figure 1) ${ }^{28}$.

The College of Medicine Research and Ethics Committee in Blantyre, Malawi and the Research Ethics Board at the Hospital for Sick Children in Toronto, Canada gave ethical approval for this study. Permission to complete the survey at the NRUs was obtained from each of the respective District Health Officers or hospital directors. Survey participants were eligible if they were healthcare personnel employed at the NRU at the time of the survey and were aware of treatment protocols at the NRU according to the District Health Officers or hospital directors. Before partaking in the survey, participants were asked to give written informed consent. This procedure was performed by the enumerator in a private setting inside or near the NRU. The same enumerator conducted the survey in English, with additional explanations in Chichewa as necessary, and each survey lasted under 30 minutes.

The survey gathered quantitative data about the NRUs including location, admission rates for SAM over the past year based on NRU documentation, and healthcare personnel working at the NRUs. Subsequent quantitative questions related to the types, frequency, and duration of psychosocial stimulation and counselling interventions as well as involvement of personnel to deliver these interventions.

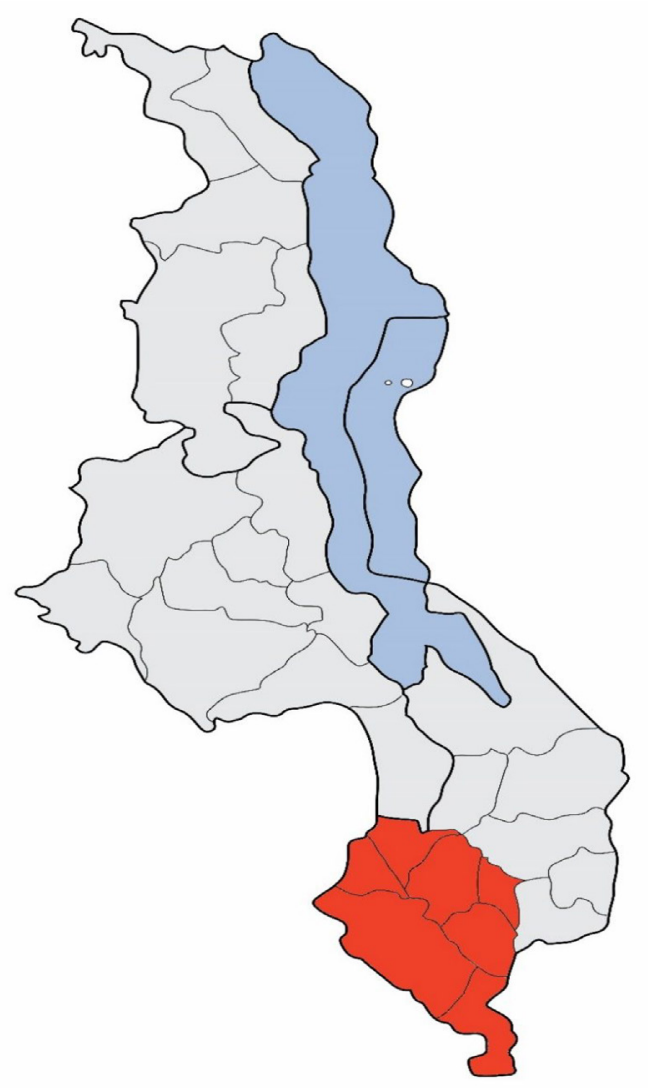

Figure 1. Map of Malawi with seven districts included in the survey highlighted

The seven districts in the survey are highlighted in red while the grey represents other districts in Malawi and the blue represents Lake Malawi

Psychosocial stimulation interventions were explained to participants as stimulation or play activities with different types of resources, materials, and exercises with the use of visual aids, audio or listening activities, play materials, physical activities, and more. If participants stated that resources were available for psychosocial stimulation, they were asked to give examples of these resources which were documented by the enumerator. There was then a question about the importance of psychosocial stimulation during children's treatment at NRUs alongside clinical and nutritional management of children using a five-point Likert scale. Participants were required to briefly justify their answers which were recorded by the enumerator as quotations.

Data from the surveys were entered into a Research Electronic Data Capture database and analyzed in Stata $14^{29,30}$. Descriptive statistics were presented as medians and interquartile ranges (IQRs) for continuous variables, and as proportions and percentages for categorical variables. Reporting guidelines for surveys were followed throughout this manuscript ${ }^{31}$.

\section{Results}

The completion rate for the in-person survey interviews was 
$16 / 16(100 \%)$ with all personnel approached at each of the respective NRUs agreeing to participate. This included NRUs in urban $(2 / 16,13 \%)$, rural $(8 / 16,50 \%)$, and semi-urban (6/16, 38\%) settings. NRUs included community-based clinics $(3 / 16,19 \%)$ as well as hospitals $(13 / 16,81 \%)$. Two $(2 / 13,15 \%)$ of the hospitals were also university hospitals and/or research sites. The number of wards including the NRUs at the various sites ranged from two to 27 wards with a median number of 5 [IQR, 5-6]. The total number of pediatric beds across the sites added up to 718 , ranging between 15 and 168 and translating to a median of 31 [IQR, 25-51]. The median admission rate over the previous year for each of the NRUs was 53 [IQR, 29-230] with a range of 10 to 393 admissions at each site, totalling 1808 admissions across the 16 NRUs (Table 2). The median number of nurses, homecraft workers, and nutritionists employed at the NRUs were 4 [IQR, 2-6], $3[\mathrm{IQR}, 2-6]$, and $0[\mathrm{IQR}, 0-1]$, respectively, for a total of 139 core healthcare personnel at all the NRUs in this survey.

Table 1. Population from the Republic of Malawi 2008 Population and Housing Census for districts included in the survey and number of NRUs within these districts ${ }^{28}$

\begin{tabular}{|l|l|l|l|}
\hline District & Population & $\begin{array}{l}\text { Number of } \\
\text { NRUs }\end{array}$ & $\begin{array}{l}\text { Population } \\
\text { relative to } \\
\text { NRUs }\end{array}$ \\
\hline Blantyre & 999,491 & 2 & 499,746 \\
\hline Chikwawa & 438,895 & 3 & 146,298 \\
\hline Chiradzulu & 290,946 & 3 & 96,982 \\
\hline Mwanza & 94,476 & 1 & 94,476 \\
\hline Neno & 108,897 & 1 & 108,897 \\
\hline Nsanje & 238,089 & 3 & 79,363 \\
\hline Thyolo & 587,255 & 3 & 195,818 \\
\hline Total & $\mathbf{2 , 7 5 8 , 2 4 8}$ & $\mathbf{1 6}$ & $\mathbf{1 7 2 , 3 9 1}$ \\
\hline
\end{tabular}

Table 2. SAM admissions and healthcare personnel at the NRUs across the seven districts included in the survey

\begin{tabular}{|l|l|l|l|l|}
\hline District & $\begin{array}{l}\text { SAM } \\
\text { admissions } \\
\text { over the } \\
\text { previous } \\
\text { year }\end{array}$ & Nurses & $\begin{array}{l}\text { Homecraft } \\
\text { workers }\end{array}$ & Nutritionists \\
\hline Blantyre & 370 & 11 & 9 & 2 \\
\hline Chikwawa & 150 & 10 & 19 & 1 \\
\hline Chiradzulu & 158 & 13 & 7 & 0 \\
\hline Mwanza & 320 & 4 & 3 & 2 \\
\hline Neno & 25 & 6 & 2 & 0 \\
\hline Nsanje & 318 & 8 & 15 & 1 \\
\hline Thyolo & 467 & 15 & 10 & 1 \\
\hline Total & $\mathbf{1 8 0 8}$ & $\mathbf{6 7}$ & $\mathbf{6 5}$ & $\mathbf{7}$ \\
\hline
\end{tabular}

None of the NRUs included in this survey have protocols for providing psychosocial stimulation. Half of participants $(8 / 16,50 \%)$ answered that children are involved in some kind of psychosocial stimulation during inpatient treatment at NRUs. As with counselling, nurses and/or homecraft workers are involved in delivery of this type of intervention. One NRU located at a research hospital in Blantyre involves caregivers and children psychosocial stimulation between 60 and 120 minutes per week, though this NRU is conducting a trial evaluating an intervention with psychosocial stimulation and therefore nurses have been trained on Care for Child Development ${ }^{19,32}$. All other NRUs have interventions below WHO recommendations of 15 to 30 minutes per day ${ }^{4,11}$. With regards to resources for psychosocial stimulation, most NRUs have play materials $(9 / 16,56 \%)$, one NRU has visual aids $(1 / 16,6 \%)$, and none have technology for audio or listening activities. Examples of resources include dolls, toy cars, rattles, balls, drums, and blocks. Many of the NRUs have no resources at all for psychosocial stimulation $(7 / 16$, $44 \%$ ).

When asked about the importance of incorporating psychosocial stimulation interventions in NRU settings, most participants stated it as "very important" (13/16, 81\%) while the remainder reported this as "somewhat important" (3/16, 19\%). No participants rated this as "neutral", "low importance", or "not at all important". One participant that answered that psychosocial stimulation is somewhat important explained, "We just take it for granted and we don't encourage play therapy and stimulation and we don't supervise." Another participant stated, "The time that we give to the kids is limited because of shortage of staff and being too busy," and another said, "We need a practical training on stimulation and play therapy."

In addition to care provided by these staff members, every participant answered that primary caregivers are responsible for feeding, bathing, and monitoring their children throughout the admission period. All NRUs provide counselling to caregivers on nutrition topics including promotion of breastfeeding and feeding frequently with energy- and nutrient-dense foods. Most NRUs (15/16, 94\%) also conduct counselling on WASH practices. Over half of the NRUs $(9 / 16,56 \%)$ give additional counselling on topics including family planning $(7 / 16,44 \%)$, HIV management $(3 / 16,19 \%)$, cooking $(2 / 16,13 \%)$, and vegetable gardens $(2 / 16,13 \%)$. The frequency of all counselling sessions is daily for half of the NRUs $(8 / 16,50 \%)$, every two days for a quarter of the NRUs (4/16, 25\%), or once per week $(2 / 16,13 \%)$ or at admission and discharge only $(2 / 16,13 \%)$. Most participants reported counselling sessions of 15 to 30 minutes $(10 / 16,63 \%)$ and the remainder have sessions which are below 15 minutes $(6 / 16,38 \%)$. All but one of the NRUs typically do individual or small group counselling sessions $(15 / 16,94 \%)$ as opposed to larger group sessions. These counselling sessions are led by nurses and/or homecraft workers. Though all the NRUs deliver counselling, none distribute counselling materials for caregivers to take home at the time of their children's discharge.

\section{Discussion}

This cross-sectional interview survey of 16 NRUs in the Southern Region of Malawi provides insight about the interventions involving caregivers and their children with SAM including counselling and psychosocial stimulation. Although half of the participants of this survey reported that children are given psychosocial stimulation interventions during inpatient treatment, none of the NRUs have protocols for delivery. Results showed that counselling of caregivers on other topics is provided at all NRUs, yet these sessions are done in limited time periods and caregivers are not given counselling materials at discharge. The duration of sessions at all NRUs is also below the basic WHO recommendations 
in all cases ${ }^{11}$. Only one other study has examined the application of the WHO recommendations for SAM treatment in two NRUs in a rural area near Durban, South Africa and indicated that psychosocial stimulation was not provided and that counselling of caregivers was unstructured and spontaneous ${ }^{27}$. This aligns closely with the pattern seen in this survey. Though participants of this survey value the importance of psychosocial stimulation, these interventions might not be provided in a structured and consistent manner in many NRU settings due to a lack of supplies, training, and standardization of interventions. The availability and use of materials or media to support counselling and psychosocial stimulation are also lacking, and therefore the effectiveness of these interventions provided is likely limited ${ }^{33}$.

Results from a feasibility study evaluating a nurse-led counselling intervention for caregivers of children with SAM at Queen Elizabeth Central Hospital in Blantyre, Malawi have indicated that involvement staff members other than nurses may be important for implementation of this type of intervention. This could include personnel like homecraft workers, for instance. This feasibility study also demonstrated that consistent refresher training of intervention content is linked to motivation of staff members ${ }^{23}$. A promising result of this survey is the recognition of the importance of psychosocial stimulation for children with SAM in NRU settings irrespective of the interventions provided at the NRUs. This could indicate that NRU staff members would be willing to deliver interventions like the Kusamala Program.

An incidental finding from the survey is the substantial decline in admissions for SAM at NRUs in Malawi. For instance, in 2015 the NRU at Queen Elizabeth Central Hospital had 622 SAM admissions with a monthly bed occupancy of 52/57 $(91 \%)^{9}$. Over 2018, this number dropped substantially with a total of 360 admissions translating to a bed occupancy of $16 / 57$ (28\%). Though there appear to be fewer admissions to NRUs for SAM than in previous years, these vulnerable children still require comprehensive interventions to improve outcomes including survival, nutritional status, and development.

\section{Limitations}

A main limitation of this cross-sectional study is that the use of a quantitative, fixed-response survey. One representative was designated at each NRU which could increase risk of selection bias. Furthermore, the nature of the survey did not allow for participants to describe in detail the types of interventions provided at the NRUs and their experiences and perceptions around these interventions. This could be further explored by applying a qualitative research approach. There is also potential for social bias in this study especially with regards to the question about importance of psychosocial stimulation, yet all participants were asked to explain their answers. Lastly, this survey is not necessarily representative of all NRUs in Malawi and therefore a next step could be to conduct a larger assessment of NRUs across the country including the collection of admission data and an evaluation of quality of care and interventions.

\section{Future directions}

Following discharge from inpatient treatment at NRUs, children with SAM have a high risk of mortality, poor nutritional outcomes including stunting and recurrence of malnutrition, and inhibited development which is longlasting $^{22,24,34-37}$. Based on the results of this survey, NRUs in Malawi require additional investment in comprehensive interventions to improve outcomes in these most vulnerable children. Scale-up of interventions will require standardized protocols and basic resources to support these interventions including play and counselling materials. Within Malawi, it will likely be feasible to train nurses, homecraft workers, and nutritionists which could be best planned when NRU admissions are lowest, typically between April and October. Though further evidence is needed to understand the most effective interventions for children hospitalized with SAM, what we do know is that these children require nurturing care which involves appropriate feeding, WASH practices, and psychosocial stimulation. Caregivers require support to provide the best care possible to their children, and NRU staff members have an opportunity to give this support to caregivers at a critical time in their children's lives.

\section{Acknowledgements}

The authors would like to thank Jacqueline Daniel for her role in graphic design of the map of Malawi displayed in this manuscript.

\section{Competing interests}

None declared.

\section{Funding}

Funding for this survey was provided by Grand Challenges Canada (1707-07564). Grand Challenges Canada is funded by the Government of Canada and is dedicated to supporting Bold Ideas with Big Impact $\AA^{\circledR}$. The funder had no role in designing this study, carrying out study activities or analysis, or writing the manuscript.

\section{Authors' contributions}

AID, WV, MvdH, MG, EM, and RHJB conceived and designed the survey and protocol. AID and $\mathrm{KC}$ collected the data. AID performed the statistical analysis. AID, KC, IP, $\mathrm{MB}$, and RHJB contributed to interpretation of data. AID drafted the manuscript. All authors were involved in critical review of the manuscript and approved the final manuscript.

\section{References}

1. UNICEF/WHO/World Bank Group. Levels and Trends in Child Malnutrition. 2017. https://data.unicef.org/wp-content/ uploads/2017/05/JME-2017-brochure.pdf. Accessed November 1, 2018.

2. World Health Organization. Community-Based Management of Severe Acute Malnutrition: A Joint Statement by the World Health Organization, the World Food Programme, the United Nations System Standing Committee on Nutrition and the United Nations Children's Fund.; 2007. https://www.who.int/nutrition/topics/Statement community_based_man_sev_acute_mal_eng.pdf?ua $=1$. Accessed November 1, 2018.

3. World Health Organization. WHO Child Growth Standards and the Identification of Severe Acute Malnutrition in Infants and Children. Geneva; 2009. https://apps.who.int/iris/bitstream/ handle/10665/44129/9789241598163 eng.pdf?ua=1. Accessed November 1, 2018.

4. World Health Organization. Guideline: Updates on the Management of Severe Acute Malnutrition in Infants and Children. Geneva; 2013. http://www.ncbi.nlm.nih.gov/books/NBK190317/. Accessed November 1, 2018.

5. National Statistical Office/Malawi ICF. Malawi Demographic and Health Survey 2015-16. Zomba, Malawi: National Statistical Office and ICF; 2017. http://dhsprogram.com/pubs/pdf/FR319/FR319.pdf. Accessed November 1, 2018. 
6. Kathumba S. Creating an enabling policy environment for effective CMAM implementation in Malawi. Field Exchange 43: Government experiences of CMAM scale up. http://www.ennonline.net/fex/43/ creating. Published 2012. Accessed November 1, 2018.

7. Malawi Ministry of Health. National CMAM Operational Plan 2017 2021. Lilongwe; 2016. https://www.fantaproject.org/sites/default/files/ resources/Malawi-CMAM-Operational-Plan-Dec2016.pdf. Accessed November 1, 2018.

8. Concern Worldwide. Scaling Up Community-Based Management of Acute Malnutrition: Implementing the CAS Program in Malawi. Lilongwe; 2013. Accessed November 1, 2018.

9. Kouam E. Evaluation of Community Management of Acute Malnutrition (CMAM) in Malawi.; 2016. https://www.unicef org/evaldatabase/files/Malawi_CMAM_Evaluation_Report Malawi_2016-002.pdf. Accessed November 1, 2018.

10. Malawi Ministry of Health. Malawi Guidelines for CommunityBased Management of Acute Malnutrition, 2nd Edition.; 2016. https:// www.fantaproject.org/sites/default/files/resources/Malawi-CMAMGuidelines-Dec2016.pdf. Accessed November 1, 2018.

11. Ashworth A, Khanum S, Jackson A, Schofield C. Guidelines for the Inpatient Treatment of Severely Malnourished Children. (World Health Organization, ed.). Geneva; 2003.

12. Kerac M, Mcgrath M, Grijalva-Eternod C, Bizouerne C, Saxton J, Bailey H, Wilkinson C, Hirsch J, Blencowe H, Shoham J, Seal A. Management of Acute Malnutrition in Infants (MAMI) Project Technical Review: Current Evidence, Policies, Practices \& Programme Outcomes. 2010.

13. Aboud FE, Yousafzai AK, Nores M. State of the science on implementation research in early child development and future directions. Ann N Y Acad Sci. 2018;1419(1):264-271. doi:10.1111/ nyas. 13722 .

14. Aboud FE, Yousafzai AK. Global health and development in early childhood. Annu Rev Psychol. 2015;66:433-457. doi:10.1146/annurevpsych-010814-015128.

15. World Health Organization, United Nations Children's Fund, World Bank Group. Nurturing Care for Early Childhood Development: Linking Survive and Thrive to Transform Health and Human Potential. Geneva; 2018. http://apps.who.int/iris/bitstream/hand le/10665/272603/9789241514064-eng.pdf?ua=1. Accessed November $1,2018$.

16. Pérez-Escamilla R, Moran VH. The role of nutrition in integrated early child development in the 21st century: contribution from the Maternal and Child Nutrition journal. Matern Child Nutr. 2017;13(1):e12387. doi:10.1111/mcn.12387.

17. Engle PL, Black MM, Behrman JR, Cabral de Mello M, Gertler PJ, Kapiriri L, Martorell R, Young ME, International Child Development Steering Group, Mello Cabral M, Gertler PJ, Kapiriri L, Martorell R, Young ME. Strategies to avoid the loss of developmental potential in more than 200 million children in the developing world. Lancet. 2007;369(9557):229-242. doi:10.1016/S0140-6736(07)60112-3.

18. Daelmans B, Black MM, Lombardi J, Lucas J, Richter L, Silver K, Britto P, Yoshikawa H, Perez-Escamilla R, MacMillan H, Dua T, Bouhouch RR, Bhutta Z, Darmstadt GL, Rao N. Effective interventions and strategies for improving early child development. BMJ. 2015;351:h4029. doi:10.1136/bmj.h4029.

19. World Health Organization. Care for Child Development Package.; 2012.http://www.unicef.org/earlychildhood/index_68195.html. Accessed November 1, 2018.

20. Van den Heuvel M, Voskuil W, Chidzalo K, Kerac M, Reijneveld SA, Bandsma R, Gladstone M. Developmental and behavioural problems in children with Severe Acute Malnutrition in Malawi: A cross-sectional study. J Glob Health. 2017;7(2):1-10. doi:10.7189/jogh.07.020416.

21. Grantham-McGregor S, Powell C, Walker S, Chang S, Fletcher
P. The long-term follow-up of severely malnourished children who participated in an intervention program. Child Dev. 1994;65(2 Spec No):428-439.

22. Kerac M, Bunn J, Chagaluka G, Bahwere P, Tomkins A, Collins S, Seal A. Follow-up of post-discharge growth and mortality after treatment for severe acute malnutrition (FuSAM study): A prospective cohort study. PLoS One. 2014;9(6):1-10. doi:10.1371/journal.pone.0096030.

23. Daniel AI, van den Heuvel M, Gladstone M, Bwanali M, Voskuijl W, Bourdon C, Potani I, Fernandes S, Njirammadzi J, Bandsma RHJ. A mixed methods feasibility study of the Kusamala Program at a nutritional rehabilitation unit in Malawi. Pilot Feasibility Stud. 2018;4(1):151. doi:10.1186/s40814-018-0347-8.

24. Moisi JC, Gatakaa H, Berkley JA, Maitland K, Mturi N, Newton CR, Njuguna P, Nokes J, Ojal J, Bauni E, Tsofa B, Peshu N, Marsh K, Williams TN, Scott JAG. Excess child mortality after discharge from hospital in Kilifi, Kenya: a retrospective cohort analysis. Bull World Health Organ. 2011;89(10):725-732, 732A. doi:10.2471/ BLT.11.089235.

25. Daniel AI, Bandsma RH, Lytvyn L, Voskuijl WP, Potani I, van den Heuvel M. Psychosocial stimulation interventions for children with severe acute malnutrition: a systematic review. J Glob Health. 2017;7(1):1-14. doi:10.7189/jogh.07.010405.

26. Nahar B, Hamadani JDJD, Ahmed T, Tofail F, Rahman A, Huda SSNN, Grantham-McGregor SMSM, Hamadani JDJD, Huda SSNN, Nahar B, Rahman A, Tofail F, Hamadani JDJD, Ahmed T, Tofail F, Rahman A, Huda SSNN, Grantham-McGregor SMSM. Effects of psychosocial stimulation on growth and development of severely malnourished children in a nutrition unit in Bangladesh. Eur J Clin Nutr. 2009;63(6):725-731. doi:10.1038/ejen.2008.44.

27. Karaolis N, Jackson D, Ashworth a., Sanders D, Sogaula N, McCoy D, Chopra M, Schofield C. WHO guidelines for severe malnutrition: are they feasible in rural African hospitals? Arch Dis Child. 2007;92(3):198204 7p. doi:10.1136/adc.2005.087346.

28. National Statistical Office. Republic of Malawi 2008 Population and Housing Census. Zomba, Malawi; 2008. http://www.mw.one. un.org/wp-content/uploads/2014/04/Malawi-Population-and-HousingCensus-Preliminary-Report-2008.pdf. Accessed November 1, 2018.

29. Harris PA, Taylor R, Thielke R, Payne J, Gonzalez N, Conde JG. Research electronic data capture (REDCap)-A metadata-driven methodology and workflow process for providing translational research informatics support. J Biomed Inform. 2009;42(2):377-381. doi:10.1016/j.jbi.2008.08.010.

30. StataCorp. Stata Statistical Software: Release 14. 2015.

31. Bennett C, Khangura S, Brehaut JC, Graham ID, Moher D, Potter BK, M. Grimshaw J. Reporting Guidelines for Survey Research: An Analysis of Published Guidance and Reporting Practices. Jewkes R, ed. PLoS Med. 2011;8(8):e1001069. doi:10.1371/journal.pmed.1001069.

32. Daniel AI, van den Heuvel M, Voskuijl WP, Gladstone M, Bwanali M, Potani I, Bourdon C, Njirammadzi J, Bandsma RHJJ. The Kusamala Program for primary caregivers of children 6-59 months of age hospitalized with severe acute malnutrition in Malawi: study protocol for a cluster-randomized controlled trial. Trials. 2017;18(550):1-11. doi:10.1186/s13063-017-2299-3.

33. Briscoe C, Aboud F. Behaviour change communication targeting four health behaviours in developing countries: A review of change techniques. Soc Sci Med. 2012;75(4):612-621. doi:10.1016/j. socscimed.2012.03.016.

34. O'Sullivan NP, Lelijveld N, Rutishauser-Perera A, Kerac M, James P. Follow-up between 6 and 24 months after discharge from treatment for severe acute malnutrition in children aged 6-59 months: A systematic review. van Wouwe JP, ed. PLoS One. 2018;13(8):e0202053. doi:10.1371/journal.pone.0202053.

35. Lelijveld N, Jalloh AA, Kampondeni SD, Seal A, Wells JC, Goyheneix M, Chimwezi E, Mallewa M, Nyirenda MJ, Heyderman RS, 
Kerac M. Brain MRI and cognitive function seven years after surviving an episode of severe acute malnutrition in a cohort of Malawian children. 2018. doi:10.1017/S1368980018003282.

36. Prado EL, Dewey KG. Nutrition and brain development in early life. Nutr Rev. 2014;72(4):267-284. doi:10.1111/nure.12102.
37. Lenters LM, Wazny K, Webb P, Ahmed T, Bhutta ZA. Treatment of severe and moderate acute malnutrition in low- and middle-income settings: a systematic review, meta-analysis and Delphi process. BMC Public Health. 2013;13 Suppl 3:S23. doi:10.1186/1471-2458-13S3-S23. 